\title{
Gout, hyperuricemia and cardiovascular risk
}

\author{
Laura Morbidoni, ${ }^{1}$ Diletta Olivari ${ }^{2}$ \\ ${ }^{1}$ Ospedale Principe di Piemonte, Area Vasta 2 ASUR Marche, Senigallia (AN); ${ }^{2}$ Clinica Medica, Università Politecnica delle \\ Marche, Ancona, Italy
}

\begin{abstract}
Concern about gout-related increase in risk of hypertension and cardiovascular diseases has been raising in recent years. A similar relationship has been postulated even for asymptomatic hyperuricemia. The aims of this review are to appraise the available evidence about: i) the relationship between hyperuricemia itself and/or gout and cardiovascular diseases; ii) the effect of decreasing serum acid uric level on the rate of cardiovascular events. To meet this purpose, we did an extensive analysis of literature, limiting the search to articles in English, indexed in Medline and published in the last 17 years. Most of the retrieved studies were conducted on surrogate outcomes, whereas randomized trials on clinically relevant outcomes are few and of questionable quality. Based on the available data, we may conclude that hyperuricemia itself is a probable, although weak, risk factor for hypertension and increases the risk of nephropathy in patients with type 2 diabetes mellitus. Moreover, symptomatic gout significantly increases the risk of cardiovascular events, particularly of myocardial infarction and mainly in young-adult and people without other risk factors. Regarding the effectiveness of urate-lowering drugs in the prevention of myocardial infarction, the strongest evidence supports their use in subjects affected by gout. A probable efficacy in controlling hypertension, especially in young subjects and women, as well as in preventing nephropathy in type 2 diabetic patients has also been reported. Interestingly, allopurinol administered at doses $\leq 300 \mathrm{mg}$ /day seems to protect from myocardial infarction, hypertension, total and serious cardiovascular events; preliminary evidence suggests a protective effect of febuxostat on major adverse cardiovascular events in high-risk gouty patients.
\end{abstract}

\section{Introduction}

The prevalence of hyperuricemia, defined as serum uric acid (SUA) $>7 \mathrm{mg} / \mathrm{dL}$, in general population is currently high and has been increasing over the past years. Estimates based on data from the latest US National Health and Nutrition Examination Survey conducted in 2007-2008, showed that

Correspondence: Laura Morbidoni, Ospedale Principe di Piemonte, Area Vasta 2 ASUR Marche, via Cellini 1, 60019 Senigallia (AN), Italy.

Tel.: +39.071.79092476 - +39.071.79092237.

E-mail: lauramorbidoni@alice.it

Key words: Gout; hyperuricemia; cardiovascular risk; cardiovascular diseases; urate-lowering therapy.

Conflict of interest: the authors declare no potential conflict of interest.

Received for publication: 7 March 2018.

Revision received: 29 May 2018.

Accepted for publication: 1 June 2018.

This work is licensed under a Creative Commons Attribution NonCommercial 4.0 License (CC BY-NC 4.0).

CCopyright L. Morbidoni and D. Olivari, 2018

Licensee PAGEPress, Italy

Italian Journal of Medicine 2018; 12:190-202

doi:10.4081/itjm.2018.1011 prevalence of hyperuricemia in the US population was around $21.4 \%$ (21.1\% among men; $21.6 \%$ among women), affecting 43.3 million people. ${ }^{1}$

Moreover, $54 \%$ of these subjects were obese, $50 \%$ suffered from hypertension, $14 \%$ were diabetic, $6 \%$ had a stroke and $5 \%$ had a myocardial infarction $(65 \%$ of patients with SUA $>10 \mathrm{mg} / \mathrm{dL}$ ); these comorbidities had higher prevalence than in normouricemic patients. $^{2}$

Not surprisingly, whether gout, as well as asymptomatic hyperuricemia, may be considered coresponsible of relevant morbidities, in particular cardiovascular diseases, it has become one of the most disputed topics in Internal Medicine.

The interest in this area has been farther rekindled by a five-year Japanese cohort study which showed that healthy asymptomatic hyperuricemic patients have an increased risk of overweight/obesity (3.2 fold), hypertension (2.7 fold), chronic kidney disease (2.0 fold) and dyslipidemia (1.6 fold). ${ }^{3}$

Biological basis of a possible causal role of uric acid in atherosclerosis and other metabolic diseases are strong.

Uric acid, the final product of purine metabolism, has an antioxidant activity in the extracellular environment, but opposite effects are determined by its intracellular accumulation, that promotes oxidative stress, endothelial dysfunction, vasoconstriction and inflammation with lipid oxidation contributing to development of atheroma (Figure 1). ${ }^{4,5}$ Moreover, in 
different murine models, mice artificially maintained with high SUA levels showed many metabolic alterations, such as reduction in nitric oxide endothelial production, increase in insulin-resistance, adipocytes dysfunctions, renin-angiotensin system activation, ultimately leading to glucose intolerance, obesity and hypertension. ${ }^{6,7}$

A formal demonstration of a cause-effect relationship between hyperuricemia and cardiovascular diseases would raise important therapeutic issues in terms of indication and duration of urate-lowering treatment (ULT).

ULT is currently recommended only for patients with recurrent flares, tophi, urate arthropathy and/or kidney stones and it should be initiated close to the time of the first diagnosis of gout in patients presenting specific characteristics as young age $(<40)$ and high levels of serum acid uric $(>8.0 \mathrm{mg} / \mathrm{dL}) .{ }^{8}$

However, concern raised from biological and epidemiological evidence already led the European League Against Rheumatism (EULAR) to recommend, in the 2016 Guideline about the management of gout that Every person with gout should be systematically screened for associated comorbidities and cardiovascular risk factors, including renal impairment, coronary heart disease, heart failure, stroke, peripheral arterial disease, obesity, hyperlipidemia, hypertension, diabetes and smoking, which should be addressed as an integral part of the management of gout. ${ }^{8}$

The present article would address the following questions: i) Is hyperuricemia in gouty or asymptomatic patients an independent risk factor for cardiovascular diseases or this association is due to confounders, reverse causality or common etiological factors? ii) Is hyperuricemia in gouty or asymptomatic patients an independent risk factor for diabetes mellitus and/or nephropathy complications? iii) Is a serum uric acid reduction in asymptomatic or gouty patients correlated with a corresponding reduced risk of cardiovascular diseases?

We performed an extensive search on Medline, limited to articles in English (last 17 years) and to adult population using the following search strings ('hyperuricemia OR gout') AND ('cardiovascular diseases OR cardiovascular risk'); allopurinol AND ('hyperuricemia OR gout OR cardiovascular diseases'); febuxostat AND ('hyperuricemia OR gout OR cardiovascular diseases'). Literature scan has been completed by analysis of related articles and manual search.

\section{Is hyperuricemia in gouty or asymptomatic patients an independent risk factor for cardiovascular diseases or diabetes onset?}

Hypertension and diabetes mellitus are the most frequent risk factors for cardiovascular diseases.

Several clinical studies have been conducted to clarify whether elevated SUA may play a direct, truly independent, role in cardiovascular and metabolic

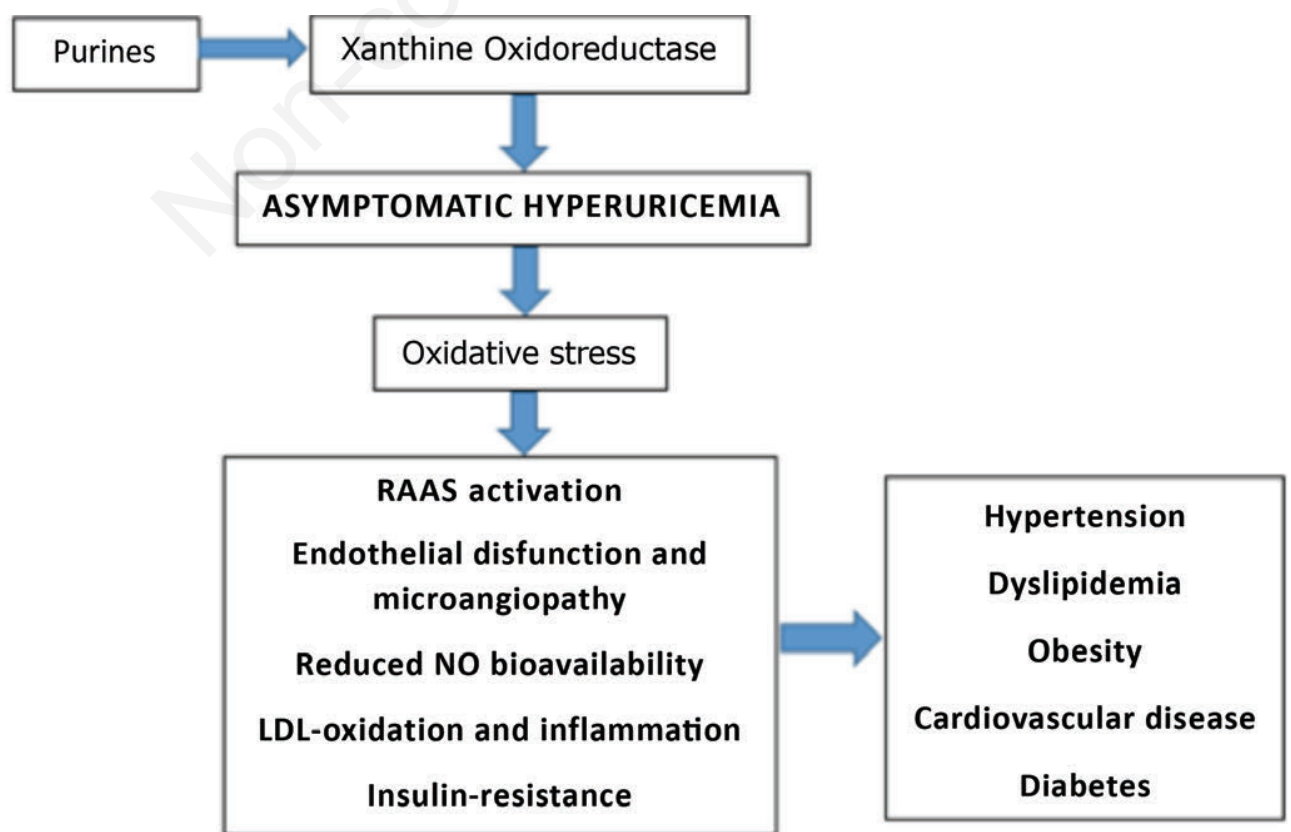

Figure 1. Biological basis of a possible causal role of uric acid in atherosclerosis and other metabolic disease. RAAS, renin-angiotensin-aldosterone system; NO, nitric oxide; LDL, low-density lipoprotein. 
diseases or observed associations have rather to be considered examples of spurious links (Tables 1 and 2).

In a crossover randomized trial ${ }^{9}$ including 30 hyperuricemic adolescents with hypertension, treatment with allopurinol was effective to lower blood pressure over one-month follow-up period; the authors concluded that at least in this specific population, uric acid may play a direct causal role on hypertension.

To test this hypothesis, a systematic review ${ }^{10}$ analyzed 18 prospective cohort studies with 55,607 adult participants. Threshold for hyperuricemia has been set (on average) at $6.2 \mathrm{mg} / \mathrm{dL}$ (range 4.6-7.0) while hypertension has been defined as blood pressure $\geq 140 / 90$ or $\geq 160 / 95 \mathrm{mmHg}$, or by the use of antihypertensive medications; subgroup analysis was performed for age, sex, and race.

With the limitation of a significant heterogeneity among studies, the meta-analysis, using adjusted or unadjusted relative risk (RR), concluded in favor of a causal relationship between hyperuricemia and hypertension, with a stronger effect in females, in younger individuals and in African American individuals.

In six studies which have managed uric acid levels as a continuous variable, the overall risk for incident hypertension raised by $13 \%$ per $1 \mathrm{mg} / \mathrm{dL}$ increase in serum uric acid level.

According to the authors, the systematic review showed a modest but significant increase of RR for incident hypertension in subjects with hyperuricemia, irrespective of the presence of other risk factors for hypertension.

Studies exploring the relationship between hyperuricemia and diabetes are conflicting. Experimental animal models have shown that uric acid could worsen insulin resistance by impairing insulin-stimulated glucose uptake, ${ }^{11}$ and two metaanalyses seem to confirm that elevated serum uric acid acts as an independent risk factor for the development of type 2 diabetes. ${ }^{12,13}$ However, a recently published Japanese prospective cohort study including 5899 patients concludes that asymptomatic hyperuricemia did not appear to be an independent risk factor for diabetes, whereas in the same setting it predicted the development of hypertension, chronic renal failure and obesity. ${ }^{3}$

Studies yielded in the past years have shown contrasting results regarding the predictive value of high uric acid levels for coronary heart diseases (CHD) and its role as an independent risk factor rather than an effect of the disease.

A meta-analysis of 16 prospective studies involving 9458 CHD cases and 155,084 controls in a comparison of individuals with serum uric acid values in the top third with those in the bottom third of the population the relative risk of CHD was 1.13 [confidence interval (CI) 1.07-1.20], with statistically significant results in male and females. In a complementary analysis of seven studies involving 6357 CHD cases and 65,978 controls which excluded individuals with known cardiovascular disease at baseline, the relative risk of CHD was 1.10 (CI 1.03-1.18). ${ }^{14}$

In eight studies with adjustment for possible confounders the odds ratio was 1.02 (CI 0.91-1.14), not statistically significant; this result decreases the likelihood that any association between serum uric acid and CHD is independent from possible confounders.

Results obtained from this meta-analysis suggest that serum uric acid levels are unlikely to be a major determinant of CHD.

A more recent systematic review including 26 prospective cohort studies and 402,997 participants has reached similar conclusion; in particular unadjusted RR for CHD incidence, based on 13 studies, was 1.34 (95\% CI 1.19-1.49) and pooled RR, based on 9 studies, adjusted for traditional risk factors for CHD, was even lower [1.09 (95\% CI 1.03-1.16)]. Furthermore, the pooled multivariate RR of incident CHD was 1.04 (95\% CI 0.90-1.17) for men and 1.07 (95\% CI 0.82-1.32) for women, both not statistically significant. ${ }^{15}$

No clear evidence of relevant increase in mortality has been seen in patients with hyperuricemia (RR fully adjusted for traditional CHD risk factors in 8 studies was 1.16, with 95\% CI 1.01-1.30).

The only remarkable difference with the previous systematic review was the statistically significant mortality risk increase in women rather than in men (RR 1.67 CI 1.30-2.04 and 1.09 CI 0.98-1.19, respectively).

To answer the question whether there is a causal association between incidence of and mortality for stroke and hyperuricemia, a systematic review ${ }^{16}$ included 15 prospective studies with 22,571 cases of stroke and 1,042,358 participants.

Participants with hyperuricemia experienced a significantly increased risk of development of stroke based on six studies (combined RR, 1.22; 95\% CI 1.02-1.46).

The pooled estimate of multivariate RRs (five studies evaluable) was 1.08 (95\% CI 0.85-1.38) among men and 1.25 among women (95\% CI 1.04-1.46).

Unlike stroke incidence, significantly higher only in hyperuricemic women, stroke-related mortality slightly increased in both sexes.

Many experts pointed out that the small size of the observed effect and the possible role of confounding factors, highlighted by the observation that the measured relative risk progressively decreases adjusting for a growing number of known risk factors 


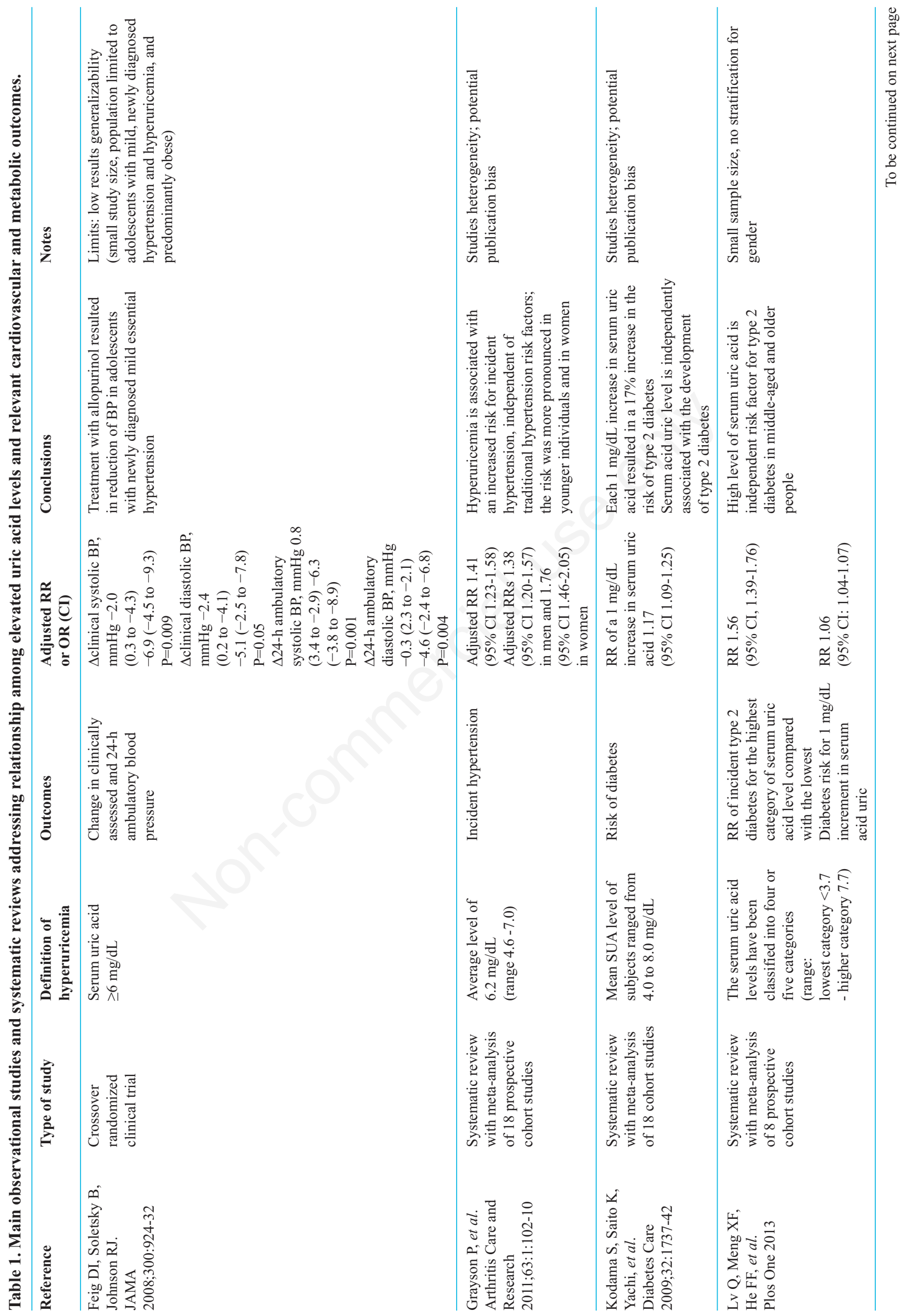




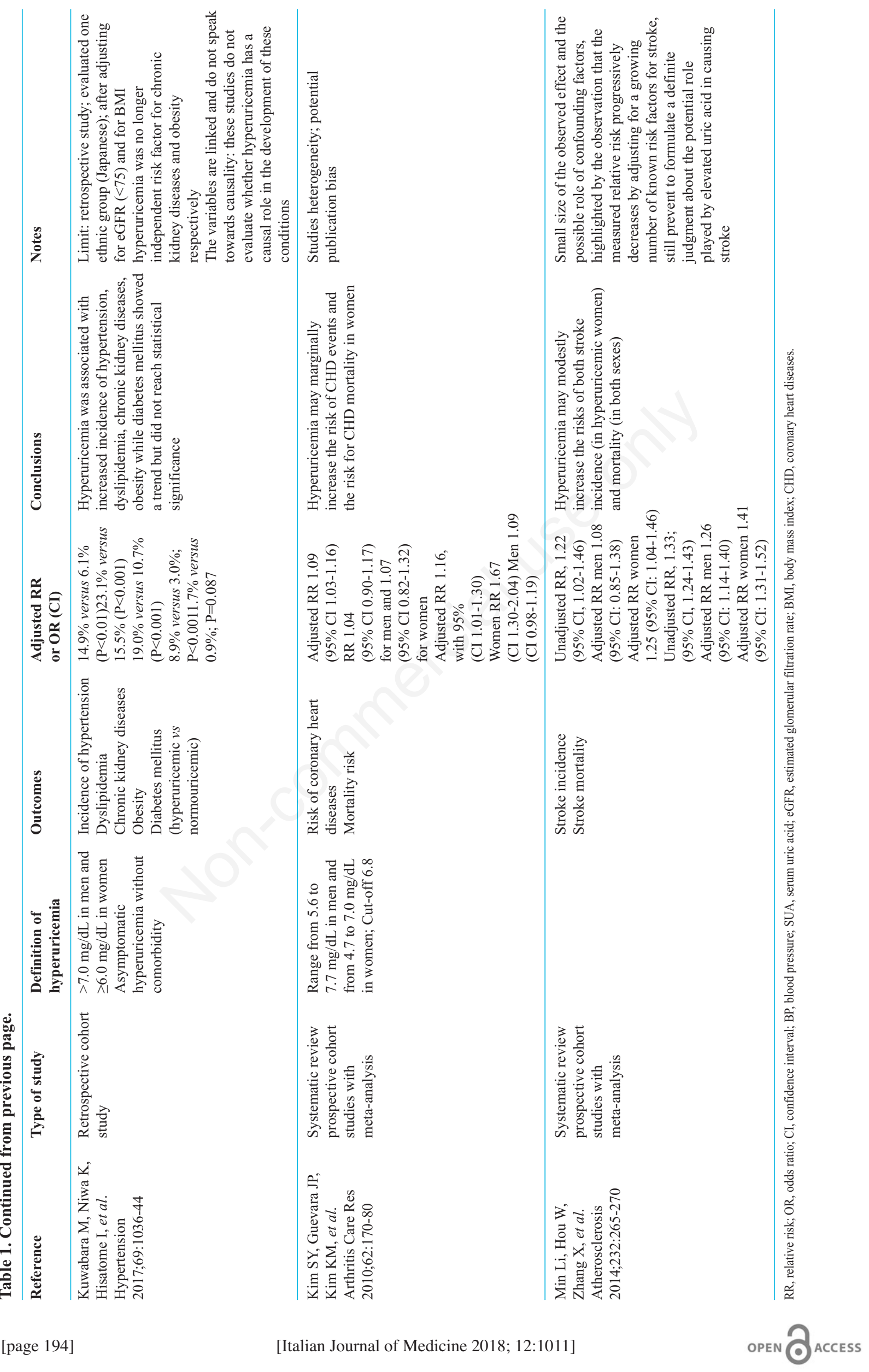


for stroke, still prevent from formulating a definite judgment about the potential role played by elevated uric acid in causing stroke.

Future studies, preferably randomized controlled trials on agents effective in lowering or preventing hyperuricemia, are needed to explore whether hyperuricemia is a potentially modifiable risk factor for stroke.

Despite the large number of observational studies and systematic reviews published, it is still not possible to provide a definitive answer to the emerging question. A lot of methodological flaws makes the global picture somewhat confusing. To cite some of them, we could list the use of different thresholds to define hyperuricemia, the choice of different outcomes variously defined and measured, the heterogeneity among studies in the selection and definition of possible confounding variables to integrate in the analysis. As a consequence, a wide dispersion in the results is evident, impairing a clear interpretation, potentially exploitable on the clinical ground. Moreover, studies incorporating higher numbers of potential risk co-factors have measured the lowest, not significant $R R$, and this finding is consistent with the negative results of a trial conducted using mendelian randomization, a technique that minimizes the confounding effects of other covariates, which has not shown an increase in the incidence of diabetes or hypertension in subjects carrying mutations that cause hyperuricemia. ${ }^{17}$

Overall, in scientific and clinical community there is a widespread sentiment that results from major meta-analyses globally suggest elevated uric acid levels per se do not confer a relevant increase in risk of ischemic events or diabetes. Whether a stronger effect could be observed in special sub-populations (e.g. women, young adults, people at low risk) it would be an interesting topic for future clinical research.

Scenario changes when we look at clinical research conducted on populations of patients with hyperuricemia, but symptomatic for gout.

Some studies reported not only that gout often occurs concurrently with myocardial infarction (MI) risk factors, but also that gouty patients have a greater risk of MI; the Framingham study, for example, found a $60 \%$ increased risk for coronary artery diseases (CAD) among gouty patients. ${ }^{18-23}$

An interesting cohort study ${ }^{24}$ included the entire population covered by National Health Institute system of Taiwan $(704,503$ individuals, aged $>20$ years); 26,556 (3.8\%) of them received a gout diagnosis, mainly men $(70 \%)$ and older than the rest of the population without gout diagnosis.

The incidence of MI was 2.20 and 0.60 per 1000 person-years among individuals with and without

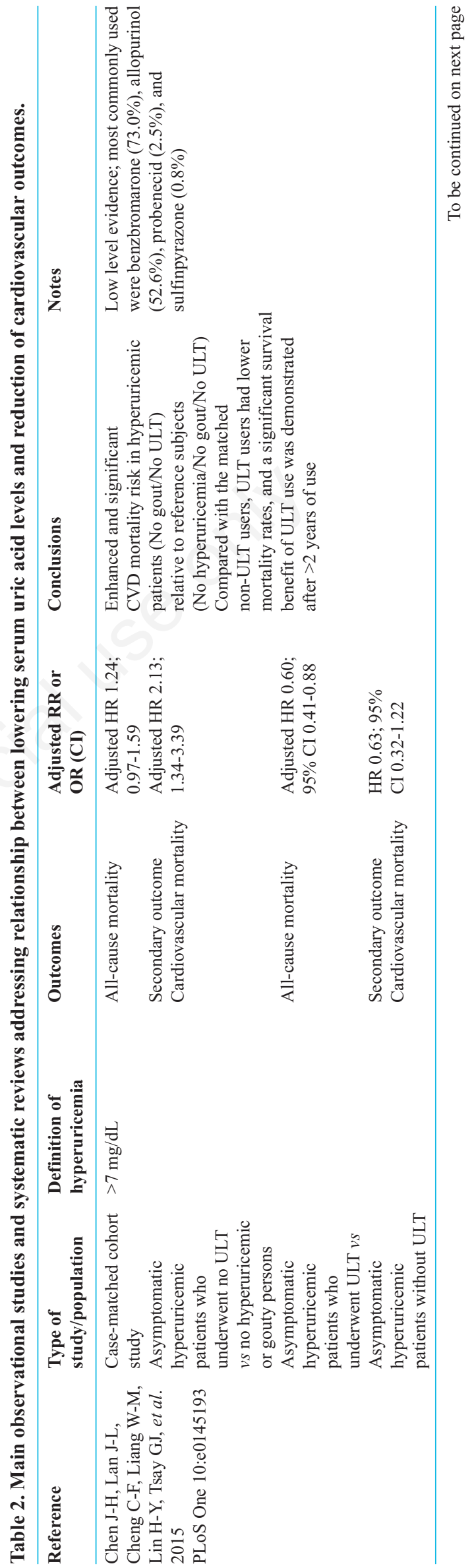




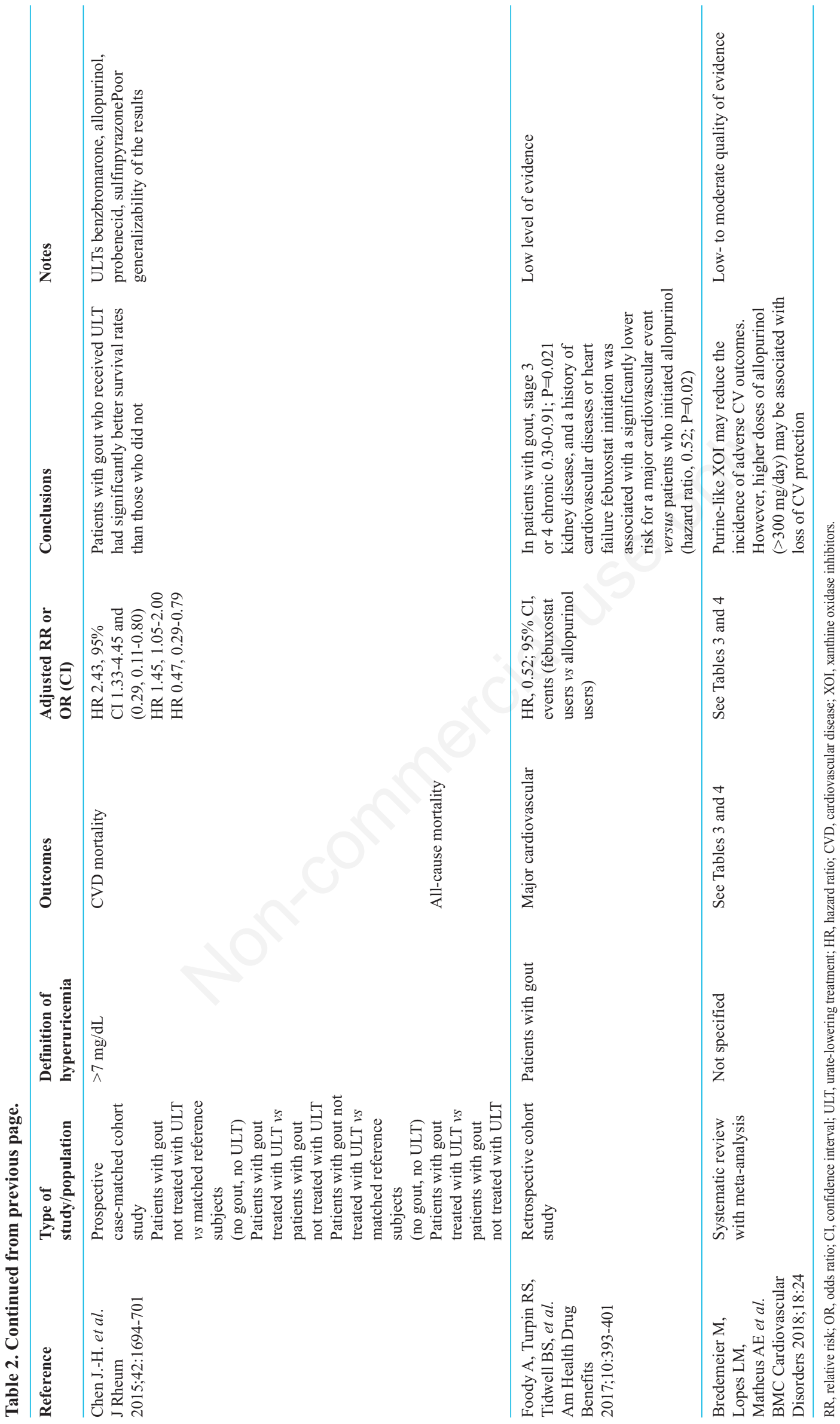


gout, respectively (log-rank test, $\mathrm{P}<0.001$ ), the incidence of both fatal and non-fatal MI was higher in gouty patients (log-rank test, $\mathrm{P}<0.001$ ), and this trend was consistent in both men and women.

After adjustment for age, sex and history of diabetes mellitus (DM), hypertension, CAD, stroke and end stage renal disease (ESRD), gout was associated with all MI and non-fatal MI, with hazard ratios (HRs) of 1.23 and 1.26 , respectively, whereas it was not an independent risk factor for fatal MI (HR 0.97).

Risk of all MI in men was slightly higher than in women.

In a total of $599,450(85.1 \%)$ individuals who did not have a history of DM, hypertension, CAD, stroke and ESRD, the risk of MI was still higher in gouty patients.

Age- and sex-adjusted HRs for the association of gout with all MI were 1.24 (95\% CI 1.10, 1.39) and $1.76(95 \%$ CI $1.45,2.13)$ for those with and without cardiovascular risk factors, respectively.

The incidence of MI was higher in individuals with gout than those without in each age group; the difference in MI incidence tended to decrease with advancing age.

Among individuals aged $>70$ years, gout was not independently associated with MI. Gout was not associated with fatal MI in any of the three age groups.

High quality data and unbiased recruitment of population are the main strength of this study, but doubts about the generalizability of results obtained in the Taiwan population to western patients have been raised.

However, results from this study strongly support the hypothesis that gout has an impact on MI risk, particularly in younger individuals and in those carrying few other specific risk factors. Proper cardiovascular monitoring should be warmly advocated for all patients who have been diagnosed with gout.

Is hyperuricemia in gouty or asymptomatic patients an independent risk factor for diabetes mellitus and/or nephropathy complications?

A recent meta-analysis ${ }^{25}$ explored the association between hyperuricemia and risk of cardiovascular diseases in a specific population of patients with type 2 diabetes mellitus (T2DM), whose risk is already higher than general population.

This meta-analysis summarizes 9 relevant studies, involving a total of more than 20,981 recruited subjects. Results indicate a significantly positive correlation between high serum uric acid levels and vascular complications [odds ratio (OR) $1.28,95 \% \mathrm{CI}$ 1.12-1.46] or mortality (HR 1.09, 95\% CI 1.03-1.16) in T2DM patients. In more detail, the increased risk of macro-vascular complications was very slight, touching the limit of the statistical significance (OR $1.03,95 \%$ CI 1.0-1.06), and peripheral vascular disease was the major determinant, whereas no trend toward an increase in cerebrovascular disease or coronary heart disease has been seen. More evident was the increased risk of microvascular complications (OR 1.47, 95\% CI 1.11-1.94), almost completely attributable to diabetic nephropathy, whose incidence almost doubled in hyperuricemic people (OR 1.91, CI 1.07-3.42).

The possibility that serum uric acid and diabetic nephropathy are non-casually related is consistent with the results of another meta-analysis ${ }^{26}$ of 21 cohort studies (sample size $=279,805$ ) showing that elevated SUA levels were an independent risk factor for incidence of kidney diseases even in general population. Moreover, several interventional trials showed beneficial effects of SUA reduction in diabetic nephropathy both in animals ${ }^{27}$ and humans. ${ }^{28}$

\section{Key messages}

- Hyperuricemia itself is a possible risk factor for hypertension. The incidence increase is moderate, more probable in obese young adults, and the exact estimate is hindered by interactions with multiple other influential factors.

- Symptomatic gout significantly increases the risk of cardiovascular events, particularly in women, young people and those without other risk factors.

- Asymptomatic hyperuricemia does not appear to significantly increase the risk of infarction or stroke. It is possible that greater influence may occur in women or young people. The exact assessment of the weight exerted by hyperuricemia per se on the risk of events is strongly hindered by the interaction with other known risk factors.

- Hyperuricemia increases the risk of nephropathy in patients with type 2 diabetes mellitus.

\section{Is a serum uric acid reduction in asymptomatic or gouty patients correlated with a corresponding reduced risk of cardiovascular diseases?}

\section{Urate-lowering therapy and hypertension}

A recently updated Cochrane Systematic Review, ${ }^{7}$ having as objective whether uric acid lowering therapy reduces blood pressure in patients with primary hypertension or prehypertension compared with placebo, concluded that currently available data from randomized controlled trials (RCTs) are insufficient to know whether ULT also lowers blood pressure and that more studies are needed. 
Pooled results indicate that ULT were not effective in reducing systolic and diastolic blood pressure measured by 24 -h continuous monitoring.

Pooled results for systolic and diastolic blood pressure measured in clinical practice included only two trials conducted in the same institution, comprising a population of adolescents with prehypertension or newly diagnosed stage 1 hypertension. In this specific setting, ULT significantly reduced systolic blood pressure. Moreover, the sub-analysis of systolic and diastolic blood pressure data, resulting from 24-h monitoring in this population of adolescents confirms a significant efficacy of urate-lowering treatment. These results, although promising, should be interpreted cautiously, because of the low-quality evidence.

\section{Urate-lowering therapy and cardiovascular diseases in persons not symptomatic for gout}

Most of the studies published so far, in order to answer this question, have investigated the effect of allopurinol, febuxostat or uricosuric agents, ${ }^{29-31}$ on surrogate outcomes of cardiovascular diseases in subjects with asymptomatic hyperuricemia. Activation of the renin-angiotensin system, endothelial function, left ventricular mass have been the most represented end-points.

In a $\mathrm{RCT}^{32}$ sixty-five patients with documented coronary artery disease, a positive exercise tolerance test, and stable chronic angina pectoris (for at least 2 months), were randomized to allopurinol (600 $\mathrm{mg}$ per day) or placebo for 6 weeks to explore time to ST depression during an exercise test as primary endpoint, and total exercise time and time to chest pain as secondary endpoints.

Results demonstrated that allopurinol at very unusual high doses was effective on these surrogate end-points, but no benefit has been found on more clinically relevant outcomes as number of symptomatic attacks or mortality (with the limit of the low statistic power). However no adverse effects of treatment were reported.

A further recent $\mathrm{RCT}^{33}$ evaluated whether allopurinol could delay the development of leg pain in patients with peripheral arterial diseases randomized to receive either allopurinol $300 \mathrm{mg}$ twice daily or placebo for 6 months. The primary outcome was the change in exercise capacity on treadmill testing at 6 months. There was a significant reduction in uric acid levels in the 23 participants receiving active treatment, but no significant change neither in the pain-free or the maximum walking distance.

The lack of randomized clinical trials conducted on clinically relevant hard outcomes makes conclusions about efficacy of urate-lowering therapies on decreasing the risk of cardiovascular ischemic diseases in patients at high risk with asymptomatic hyperuricemia, very uncertain.

However, the question remains a hot topic, characterized by an urgent need of well-conducted clinical trials. Indeed, an increased risk of mortality in patients with not-treated asymptomatic hyperuricemia has been reported. A retrospective case-matched cohort study ${ }^{34}$ analyzed all-cause and cardiovascular disease-related mortality in untreated asymptomatic hyperuricemic patients who did not receive uratelowering therapy together with the impact of ULT on reducing the risk.

In this retrospective case-matched cohort study (mean follow-up 6.4 years), 40,118 Taiwanese individuals who had never used urate-lowering therapy and who had never had gout were examined. Mortality rate was compared between 3088 hyperuricemic patients who did not receive ULT and reference subjects (no hyperuricemia, no gout, no ULT) matched for age and sex and between 1024 hyperuricemic patients who received ULT and 3,088 hyperuricemic patients who did not receive it.

After adjustment, hyperuricemic patients who did not receive urate-lowering therapy had increased risks of all cause (hazard ratio, 1.24; 0.97-1.59) and cardiovascular $(2.13 ; 1.34-3.39)$ mortality relative to the matched reference subjects. Hyperuricemic patients treated with urate-lowering therapy had a lower risk of all-cause death $(0.60 ; 0.41-0.88)$ in comparison with those who did not receive therapy.

Publication of the results from two important clinical trials is expected within 2018-2019. The first $\mathrm{RCT}^{35}$ aims to evaluate preventive effects of febuxostat on cerebral, cardiovascular, and renal events in patients with hyperuricemia compared to conventional treatment. The second trial ${ }^{36}$ is designed to explore effects of febuxostat on intima-media thickness of the carotid artery assessed by ultrasonography, a surrogate marker of cardiovascular disease risk, in patients with hyperuricemia.

Relevant data are available for the particular population of patients affected by type 2 diabetes and asymptomatic hyperuricemia. A randomized open parallel-controlled trial $^{37}$ (176 subjects randomly divided into two groups: allopurinol or conventional treatments) demonstrated that, after 3 years of treatment, allopurinol was effective in reducing not only SUA, but also urinary albumin excretion rate, serum creatinine and in increasing glomerular filtration rate. Intention-to-treat analysis indicated that the incidence of new-onset diabetic nephropathy and hypertension in the allopurinol group showed a declining trend compared to the conventional treatment group, despite a lack of significant difference.

Anyway, controversies about efficacy of ULT in 
reducing the incidence of renal complications in asymptomatic persons with elevated serum uric acid levels persist.

A systematic review ${ }^{38}$ on treatment of hyperuricemia in order to prevent gouty arthritis, renal disease, or cardiovascular events in asymptomatic patients (a total of 3 studies met the inclusion criteria) found that in asymptomatic hyperuricemic patients without renal disease, treatment resulted in increased estimated glomerular filtration rate, whereas in those with preexisting renal disease no significant elevation of serum creatinine over a 1-year follow up was seen. However, differences in renal function between the treatment and no-treatment groups were not statistically significant in any of the selected studies.

Authors concluded that there is still insufficient evidence to suggest that lowering serum uric acid level in asymptomatic patients with hyperuricemia can prevent renal disease, or cardiovascular events or even gouty arthritis.

\section{Urate-lowering therapy and cardiovascular diseases in gouty persons}

A prospective case-matched cohort study, ${ }^{39}$ recruiting 40,623 Taiwanese individuals, compared mortality rate between 1189 patients with gout who did not receive uric acid lowering therapy and reference subjects (no gout, no uric acid lowering therapy) matched for age, sex and the index date of gout diagnosis and between 764 gouty patients who received uric acid lowering therapy and 764 gouty patients who did not receive uric acid lowering therapy.

After adjustment, patients with gout not treated with uric acid lowering therapy had an increased risk of cardiovascular mortality (HR 2.43, 1.33-4.45) and all-cause mortality $(1.45,1.05-2.00)$ compared to the matched reference subjects (no gout, no uric acid lowering therapy). Patients with gout treated with uric acid lowering therapy had a lower risk of cardiovascular diseases $(0.29,0.11-0.80)$ and all-cause mortality $(0.47,0.29-0.79)$ compared to patients with gout not treated. This survival benefit was measured both in patients receiving allopurinol and benzbromarone.

Patients with gout who received uric acid lowering therapy had significantly better survival rates than those who did not. Thus, under-treatment of gout may have serious negative consequences.

The efficacy of ULT in terms of mortality reduction increases along with the treatment duration.

A retrospective cohort study ${ }^{40}$ including 2426 patients (370 receiving febuxostat and 2056 receiving allopurinol) with gout, stage 3 or 4 chronic kidney disease, and a history of cardiovascular diseases or heart failure had 162 major cardiovascular events
(3.8\% in those receiving febuxostat $v s 7.2 \%$ in those receiving allopurinol; $\mathrm{P}=0.015$ ).

Febuxostat initiation was associated with a significantly lower risk of a major cardiovascular event than patients treated with allopurinol (hazard ratio, 0.52; $\mathrm{P}=0.02$ ), mainly due to lower peripheral vascular disease-specific events $(\mathrm{P}=0.026)$.

A recent systematic review with meta-analysis, ${ }^{41}$ including 11,861 patients, compared the incidence of major adverse cardiovascular events (MACE) (e.g., cardiovascular death, non-fatal myocardial infarction, unstable angina requiring urgent revascularization or non-fatal stroke), mortality, total cardiovascular events (TCE) (e.g., cardiovascular death, acute ischemic heart disease, stroke, new or worsening hypertension, heart failure or worsening heart failure, cardiac arrhythmias, venous and arterial visceral or peripheral thrombotic events), and serious adverse events (those requiring urgent medical procedures and/or hospitalization, life-threatening or leading to death) in RCTs testing xanthine oxidase inhibitors (XOI) against placebo or no treatment.

The assumption of XOI was a sufficient criterion to be included in the meta-analysis without knowing whether the patient was hyperuricemic and asymptomatic or affected by gouty arthritis.

XOI did not significantly reduce risk of MACE $(\mathrm{OR}=0.71,0.46-1.09)$ and death $(0.89,0.59-1.33)$, but reduced risk of total cardiovascular events (TCE 0.66; 0.54 to 0.80$)$, serious adverse events $(0.64 ; 0.51$ to 0.81 ) and hypertension $(0.54,0.37$ to 0.80$)$ (Tables 3 and 4). ${ }^{41}$ XOI protected from MACE patients with previous ischemic events $(0.42,0.23-0.76)$.

Subgroup analysis showed a reduced risk of MACE in individuals who presented acute ischemic encephalic or coronary events $\left(0.42,0.23\right.$ to $0.76, \mathrm{P}=0.004, \mathrm{I}^{2}=0 \%$ $\mathrm{P}=0.914$ ). In subgroup analysis, serious cardiovascular events were more strongly reduced in studies including only patients with previous ischemic events $(0.36,0.20$ to $0.63, \mathrm{P}<0.001$ ).

Studies testing non-purine like XOI demonstrated no statistically significant cardiovascular protective effect, but confidence intervals were wide.

Quite surprisingly, meta-regression showed an association between the increase in doses of allopurinol and a higher risk of TCE and serious TCE $(\mathrm{P}<0.05)$; accordingly, lower doses (100-300 mg/day) of allopurinol were associated with lower risk of TCE. Non-purine-like XOI did not significantly modify the risk of adverse cardiovascular events, but confidence intervals were wide. Evidence quality was generally low to moderate.

Overall, urate-lowering therapy, in particular allopurinol, when administered for long time (at least more than two years) can likely help to improve cardiovascular prognosis of gouty patients. 
However, although Allopurinol is usually well tolerated, about $1-5 \%$ of patients dicontinues treatment because of an adverse reaction. The most frequent allopurinol-related side effects are nausea, diarrhea, gout flares, fever, pruritus and mild skin reactions, whereas less common are vomiting, hepatitis, agranulocytosis and headache. ${ }^{42}$

Furthermore, allopurinol is among the main causes, in Asian and Western countries, of lifethreatening severe cutaneous adverse hypersensitivity reactions (SCARs) such as Stevens-Johnson syndrome, toxic epidermal necrolysis and DRESS syndrome. Allopurinol-induced SCARs $(0.4 \%$ of treated patients) have a high mortality rate $(9-32 \%)$ due to multiorgan failure or infections. ${ }^{43}$

Main risk factors identified for allopurinol-induced

Table 3. Cardiovascular adverse events in patients treated with urate-lowering treatment (vs placebo) as shown in a systematic review by Bredemeier et al. ${ }^{41}$

\begin{tabular}{|c|c|}
\hline Primary outcomes & OR (95\% CI), P value, $I^{2}$ (P value), number of studies \\
\hline Major adverse cardiovascular events & $\begin{array}{l}0.71(0.46 \text { to } 1.09) \\
\mathrm{P}=0.120, \mathrm{I}^{2}=10 \% \\
(\mathrm{P}=0.324), 81 \text { studies }\end{array}$ \\
\hline Death & $\begin{array}{l}0.89(0.59 \text { to } 1.33) \\
\mathrm{P}=0.573, \mathrm{I}^{2}=0 \% \\
(\mathrm{P}=0.704), 90 \text { studies }\end{array}$ \\
\hline Secondary outcomes & OR $\left(95 \%\right.$ CI), P value, $I^{2}$ (P value), number of studies \\
\hline New/worsening hypertension & $\begin{array}{l}0.54(0.37 \text { to } 0.80) \\
\mathrm{P}=0.002, \mathrm{I}^{2}=0 \% \\
(\mathrm{P}=0.494), 71 \text { studies }\end{array}$ \\
\hline Total cardiovascular events & $\begin{array}{l}0.66(0.54 \text { to } 0.80) \\
P<0.001, I^{2}=49 \% \\
(P=0.002), 81 \text { studies; } \\
D-L: 0.60(0.44 \text { to } 0.82) \\
P=0.001, I^{2}=41 \% \\
(P=0.012)\end{array}$ \\
\hline Serious cardiovascular events & $\begin{array}{l}0.64(0.51 \text { to } 0.81) \\
\mathrm{P}<0.001, \mathrm{I}^{2}=34 \% \\
(\mathrm{P}=0.050), 81 \text { studies; } \\
\mathrm{D}-\mathrm{L}: 0.64(0.46 \text { to } 0.89) \\
\mathrm{P}=0.008, \mathrm{I}^{2}=24 \% \\
(\mathrm{P}=0.135)\end{array}$ \\
\hline
\end{tabular}

OR, odds ratio; $\mathrm{CI}$, confidence interval.

Table 4. Cardiovascular adverse events in patients treated with purine like urate-lowering treatment (ULT) vs non purinelike ULT, as shown in a systematic review by Bredemeier et al. ${ }^{41}$

\begin{tabular}{|c|c|c|}
\hline Primary outcomes & $\begin{array}{l}\text { Purine-like XOI (allopurinol or oxypurinol) } \\
\text { non-purine like XOI (febuxostat or topiroxostat) } \\
\text { OR ( } 95 \% \text { CI), P value, I }{ }^{2} \text { (P value), number of studies }\end{array}$ & $\begin{array}{l}\text { Non-purine like XOI } \\
\text { (febuxostat or topiroxostat) }\end{array}$ \\
\hline Major adverse cardiovascular events & $0.65(0.41$ to 1.05$), \mathrm{P}=0.076, \mathrm{I}^{2}=9 \%(\mathrm{P}=0.354), 65$ studies & $\begin{array}{l}1.13(0.40 \text { to } 3.19), \mathrm{P}=0.824 \\
\mathrm{I}^{2}=18 \%(0.290), 19 \text { studies }\end{array}$ \\
\hline Death & $0.94(0.62$ to 1.44$), \mathrm{P}=0.785, \mathrm{I}^{2}=0 \%(\mathrm{P}=0.525), 74$ studies & $\begin{array}{l}0.71(0.15 \text { to } 3.40), P=0.671, I^{2}=0 \% \\
(P=0.956), 19 \text { studies }\end{array}$ \\
\hline Secondary outcomes & $\begin{array}{l}\text { Purine-like XOI (allopurinol or oxypurinol) } \\
\text { non-purine like XOI (febuxostat or topiroxostat) } \\
\text { OR ( } 95 \% \text { CI), P value, I }{ }^{2} \text { (P value), number of studies }\end{array}$ & $\begin{array}{l}\text { Non-purine like XOI } \\
\text { (febuxostat or topiroxostat) }\end{array}$ \\
\hline New/worsening hypertension & 0.32 (0.18 to 0.58$), \mathrm{P}<0.001, \mathrm{I}^{2}=0 \%(\mathrm{P}=0.737), 55$ studies & $\begin{array}{l}0.70(0.43 \text { to } 1.12), P=0.136, I^{2}=13 \% \\
(P=0.329), 19 \text { studies }\end{array}$ \\
\hline Total cardiovascular events & $\begin{array}{l}0.57(0.46 \text { to } 0.72), \mathrm{P}<0.001, \mathrm{I}^{2}=60 \%(\mathrm{P}<0.001), 65 \text { studies; } \\
\text { D-L: } 0.48(0.31 \text { to } 0.75), \mathrm{P}=0.001, \mathrm{I}^{2}=55 \%(\mathrm{P}=0.001)\end{array}$ & $\begin{array}{l}0.90(0.62 \text { to } 1.30), \mathrm{P}=0.562, \mathrm{I}^{2}=0 \% \\
(\mathrm{P}=0.734), 19 \text { studies }\end{array}$ \\
\hline Serious cardiovascular events & $\begin{array}{l}0.59(0.46 \text { to } 0.76), \mathrm{P}<0.001, \mathrm{I}^{2}=50 \%(\mathrm{P}=0.011), 65 \text { studies; } \\
\text { D-L: } 0.56(0.36 \text { to } 0.86), \mathrm{P}=0.009, \mathrm{I}^{2}=44 \%(\mathrm{P}=0.028)\end{array}$ & $\begin{array}{l}1.04(0.58 \text { to } 1.87), P=0.901, I^{2}=0 \% \\
(P=0.967), 19 \text { studies }\end{array}$ \\
\hline
\end{tabular}

XOI, xanthine oxidase inhibitors; CI, confidence interval. 
SCARs are HLA-B58:01 carrier, female gender, older age, poor renal function or cardiovascular disease, and high allopurinol initial dosage. ${ }^{43}$

Patients with allopurinol-SCARs, switched to febuxostat, have no cross-reactivity, although two case reports showed febuxostat-induced hypersensitivity and DRESS syndrome. ${ }^{43}$

The most common febuxostat-induced adverse reactions are liver function test abnormalities, gout flares, diarrhea, headache, and musculoskeletal signs and symptoms, while nausea and vomiting, dizziness, dysgeusia, fatigue, stomach discomfort and arthralgia are less frequent. ${ }^{44}$

\section{Key messages}

- Most of the studies have been conducted on surrogate outcomes as endothelial dysfunction, ventricular hypertrophy, insulin resistance, fasting glucose level, exercise tolerance etc.; wellconducted randomized clinical trials addressing clinically relevant outcomes are desperately needed, particularly in the setting of asymptomatic patients. Observational studies, indeed, suggest that under-treatment of hyperuricemia may have serious negative consequences.

- Possible effectiveness of the urate-lowering therapy is reported: i) in improving hypertension control, especially in young subjects and in women; ii) in preventing nephropathy in type 2 diabetics.

- The strongest evidence supports the effectiveness of urate-lowering therapy in the prevention of myocardial infarction in subjects affected by symptomatic gout.

- Allopurinol ( $\leq 300 \mathrm{mg} /$ day) seems to protect from myocardial infarction, hypertension, total and serious cardiovascular events, but higher doses were associated with increased risk.

- Only conflicting results are currently available about the comparison between purine-like XOI and not-purine-like XOI efficacy on cardiovascular outcomes.

\section{Conclusions}

Hyperuricemia itself is a possible risk factor for hypertension and increases the risk of nephropathy in patients with type 2 diabetes mellitus, whereas it does not appear to significantly increase the risk of infarction, stroke, chronic renal failure or diabetes.

The exact assessment of the weight exerted by hyperuricemia per se on the risk of cardiovascular or metabolic adverse events is strongly hindered by the interaction with other known risk factors.

In hyperuricemic patients with symptomatic gout the risk of cardiovascular events, above all myocardial infarction, significantly increases, particularly in young people and those without other risk factors.

It is likely that benefits of urate-lowering therapy outweigh risks and costs in gouty patients at high risk of cardiovascular diseases and maybe in hyperuricemic diabetic patients (to protect from diabetic nephropathy) or in obese adolescents affected by arterial hypertension.

In all other cases the benefit-risk ratio is at least uncertain.

Further clinical studies are needed. They should include homogeneous patients with hyperuricemia with or without gout, compare drugs acting with different mechanisms and evaluate clinically relevant hard outcomes.

\section{References}

1. Zhu Y, Pandya BJ, Choi HK. Prevalence of gout and hyperuricemia in the US general population: the NHANES 2007-2008. Arthrit Rheum 2011;63:3136-41.

2. Zhu Y, Pandya BJ, Choi HK. Comorbidities of gout and hyperuricemia in the US general population: NHANES 2007-2008. Am J Med 2012;125:679-87.e1.

3. Kuwabara M, Niwa K, Hisatome I, et al. Asymptomatic Hyperuricemia without comorbidities predicts cardiometabolic diseases, five year Japanese cohort study. Hypertension 2017;69:1036-44.

4. Feig DI. Uric acid and cardiovascular risk. N Engl J Med 2008;359:1811-21.

5. Biscaglia S. Uric acid and coronary artery disease: An elusive link deserving further attention. Int J Cardiol 2015 [Epub ahead of print].

6. Karnbay E. Uric acid in metabolic syndrome: from an innocent bystander to a central player. Eur J Intern Med 2016;29:3-8.

7. Gois PHF. Pharmacotherapy for hyperuricemia in hypertensive patients. Cochrane Database Syst Rev 2017;4:CD008652.

8. Richette P, Doherty M, Pascual E, et al. 2016 updated EULAR evidence-based recommendations for the management of gout. Ann Rheum Dis 2016;0:1-14.

9. Feig DI, Soletsky B, Johnson RJ. Effect of allopurinol on blood pressure of adolescents with newly diagnosed essential hypertension: a randomized trial. JAMA 2008;300:924-32.

10. Grayson P. Hyperuricemia and incident hypertension: a systematic review and meta-analysis. Arthritis Care Res 2011;63:102-10.

11. Tassone EJ, Presta I, Sciacqua A, et al. Uric acid promotes endothelial dysfunction: A new molecular model of insulin resistance. Eur $\mathrm{J}$ Clin Invest 2011;41:81-2.

12. Kodama S, Saito K, Yachi M, et al. Association between serum uric acid and development of type 2 diabetes. Diabetes Care 2009;32:1737-42.

13. Lv Q, Meng XF, He FF, et al. High serum uric Acid and increased risk of type 2 diabetes: a systemic review and meta-analysis of prospective cohort studies. PLoS One 2013;8:e56864. 
14. Wheeler JG, Juzwishin KDM, Eiriksdottir G, et al. Serum uric acid and coronary heart disease in 9,458 incident cases and 155,084 controls: prospective study and meta-analysis. PLoS Med 2005;2:e76.

15. Kim SY, Guevara JP, Kim KM, et al. Hyperuricemia and coronary heart disease: a systematic review and metaanalysis. Arthritis Care Res (Hoboken) 2010;62:170-80.

16. Min Li, Hou W, Zhang X, et al. Hyperuricemia and risk of stroke: a systematic review and meta-analysis.of prospective studies. Atherosclerosis 2014;232:265-70.

17. Kleber ME, Delgado G, Grammer TB, et al. Uric acid and cardiovascular events: a mendelian randomization study. J Am Soc Nephrol 2015;26:2831-8.

18. Choi HK, Ford ES, Li C, Curhan G. Prevalence of the metabolic syndrome in patients with gout: the Third National Health and Nutrition Examination Survey. Arthritis Rheum 2007;57:109-15.

19. Choi HK, De Vera MA, Krishnan E. Gout and the risk of type 2 diabetes among men with a high cardiovascular risk profile. Rheumatology 2008;47:1567-70.

20. Mellen PB, Bleyer AJ, Erlinger TP, et al. Serum uric acid predicts incident hypertension in a biethnic cohort: the atherosclerosis risk in communities study. Hypertension 2006;48:1037-42.

21. Krishnan E, Baker JF, Furst DE, Schumacher HR. Gout and the risk of acute myocardial infarction. Arthritis Rheum 2006;54:2688-96.

22. Choi HK, Curhan G. Independent impact of gout on mortality and risk for coronary heart disease. Circulation 2007;116:894-900.

23. Abbott RD, Brand FN, Kannel WB, Castelli WP. Gout and coronary heart disease: the Framingham study. J Clin Epidemiol 1988;41:237-42.

24. Kuo CF, Yu KH See LC, et al. Risk of myocardial infarction among patients with Gout. A nationwide population-based study. Rheumatology 2013; 52:111-7.

25. Yili X, Jaiyu Z, Li G, et al. Hyperuricemia as an independent predictor of vascular complications and mortality in type 2 diabetes patients: a meta-analysis. PLoS One 2013;8:e78206.

26. Li YL, Wang L, Li J, Huang Y, Yuan WM. The correlation between uric acid and the incidence and prognosis of kidney diseases: a systematic review and meta-analysis of cohort studies. Zhonghua Nei Ke Za Zhi 2011;50:555-61.

27. Kosugi T, Nakayama T, Heinig M, et al. Effect of lowering uric acid on renal disease in the type 2 diabetic $\mathrm{db} / \mathrm{db}$ mice. Am J Physiol Renal Physiol 2009;297:F481-8.

28. Doria A, Krolewski AS. Diabetes: lowering serum uric acid levels to prevent kidney failure. Nat Rev Nephrol 2011;7:495-6.

29. Szwejkowski BR, Gandy SJ, Rekhraj A, et al. Allopurinol reduces left ventricular mass in patients with type II diabetes and left ventricular hypertrophy. JACC 2013;61:10.

30. Tani S, Nagao K, Hirayama K, et al. Effect of febuxostat on cardiovascular risk in hyperuricemic patients with hypertension. Clin Drug Investig 2015;35:823-31.

31. Ohta Y, Hyshi Y. Effective uric acid -lowering treatment for hypertensive patients with hyperuricemia. Hypert Res 2016;1-5.

32. Noman A, Ang DSC, Ogston S, et al. Effect of high-dose allopurinol on exercise in patients with chronic stable angina: a randomised, placebo controlled crossover trial. Lancet 2010;375:2161-7.

33. Robertson AJ, Struthers AD. A randomized controlled trial of allopurinol in patients with peripheral arterial disease. Canad J Cardiol 2016;32:190-6.

34. Chen J-H, Lan J-L, Cheng C-F, et al. Effect of uratelowering therapy on all-cause and cardiovascular mortality in hyperuricemic patients without gout: a casematched cohort study. PLoS One 2015;10:e0145193.

35. Kojima S, Matsui K, Ogawa H, et al. Rationale, design, and baseline characteristics of a study to evaluate the effect of febuxostat in preventing cerebral, cardiovascular, and renal events in patients with hyperuricemia. J Cardiol 2017;69:169-75.

36. Oyama J, Tanaka A, Sato Y, et al. Rationale and design of a multicenter randomized study for evaluating vascular function under uric acid control using the xanthine oxidase inhibitor, febuxostat: the PRIZE study. Cardiovasc Diabetol 2016;15:87.

37. Peng L. Allopurinol treatment improves renal function in patients with type 2 diabetes and asymptomatic hyperuricemia: a three years of randomized parallelcontrolled study, Clin Endocrinol (Oxf) 2015;83:475-82.

38. Vinick O, Wechalekar MD, Buchbinder R. Treatment of asymptomatic hyperuricemia for the prevention of gouty arthritis, renal disease and cardiovascular events: a systematic literature review. J Rheumatol Suppl 2014;92:70-4.

39. Chen JH, Lan JL, Cheng CF, et al. Effect of uratelowering therapy on the risk of cardiovascular disease and all-cause mortality in patients with gout: a casematched cohort study. J Rheumatol 2015;42:1694-701.

40. Foody A, Turpin RS, Tidwell BS, et al. Major cardiovascular events in patients with gout and associated cardiovascular disease or heart failure and chronic kidney disease initiating a xanthine oxidase inhibitor. Am Health Drug Benef 2017;10:393-401.

41. Bredemeier M, Lopes LM, Matheus AE, et al. Xanthine oxidase inhibitors for prevention of cardiovascular events: a systematic review and meta-analysis of randomized controlled trials. BMC Cardiovasc Disord 2018 [Epub ahead of print].

42. Keenan RT. Safety of urate-lowering therapies: managing the risks to gain the benefits. Rheum Dis Clin N Am 2012;38:663-80.

43. Wang CW. Immunopathogenesis and risk factors for allopurinol severe cutaneous adverse reactions. Curr Opin Allergy Clin Immunol 2016;16:339-45.

44. Stamp L. Safety profile of anti-gout agents: an update. Curr Opin Rheumatol 2014;6:2. 